\title{
Concordance between high antibiotic prescribing and high opioid prescribing among primary care physicians: a cross-sectional study
}

\author{
Bradley J. Langford PharmD, Cynthia Chen MSc, Nick Daneman MD MSc, Kevin A. Brown PhD, \\ Tara Gomes MHSc PhD, Jennie Johnstone MD PhD, Julie Wu MSc, Valerie Leung BScPhm MBA, \\ Gary Garber MD, Kevin L. Schwartz MD MSc
}

\section{Abstract}

Background: Antimicrobial resistance and opioid misuse both present major public health challenges, and identifying high prescribers of both of these agents can help provide a common target for intervention. We sought to determine the association between being a high prescriber of antibiotics and being a high prescriber of opioids in the primary care setting.

Methods: We performed a cross-sectional study of the antibiotic- and opioid-prescribing habits of primary care physicians in Ontario, Canada between Mar. 1, 2017, and Feb. 28, 2018, using administrative databases. We defined high prescribers as the top quartile of antibiotic or opioid prescribers using 3 antibiotic-prescribing metrics (prescriptions per patient visit, proportion of prescriptions that were broad spectrum and proportion of prescriptions $>8 \mathrm{~d}$ ) and 3 opioid-prescribing metrics (prescriptions per patients seen, proportion of prescriptions $>90 \mathrm{mg}$ of morphine equivalents and proportion of prescriptions $>28 \mathrm{~d}$ ). We tabulated agreement between prescribing metrics using the $\kappa$ statistic.

Results: We included 9994 physicians. We observed minimal overlap between high antibiotic initiation and high opioid initiation (618 physicians [6.2\%]) ( $\kappa=0.00,95 \%$ confidence interval -0.02 to 0.02$)$. There was slight agreement between the antibioticprescribing indices and between the opioid-prescribing indices (within-class, range of $\kappa 0.05$ to 0.18 ). There was slight disagreement to slight agreement across antibiotic- and opioid-prescribing metrics (between-class, range of $\kappa-0.09$ to 0.16 ).

Interpretation: Among primary care physicians, there was a lack of association between high antibiotic prescribing and high opioid prescribing. Our findings suggest that separate tailored approaches to antibiotic and opioid stewardship strategies are needed.

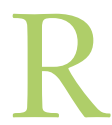

ising antimicrobial resistance is an urgent public health threat. Most antibiotics are prescribed in the outpatient setting, which acts as an important driver for antibiotic resistance in the community. ${ }^{1}$ In the United States alone, antibiotic-resistant infections claim 23000 lives annually. ${ }^{2}$ At the same time, another drug-related public health crisis looms. The epidemic of opioid overuse is evolving rapidly, and, in the US, prescription opioids are associated with 17000 overdose-related deaths annually. ${ }^{3}$ Although deaths related to nonprescribed opioids are increasing, prescription opioids still account for at least $25 \%$ of opioid-related deaths. ${ }^{4}$

Despite their obvious differences, these 2 public health threats have notable commonalities. In North America, both antibiotic overuse and opioid overuse are largely iatrogenic in origin owing to overprescribing behaviour. ${ }^{5}$ There is wide variability in prescribing practices for both of these drug classes that is not fully explained by differences in practice settings and patient populations. ${ }^{6-8}$ In addition, both patients of high prescribers of antibiotics and patients of high prescribers of opioids are more likely to experience harm than patients of low prescribers, ${ }^{6,8}$ which provides an impetus for urgent intervention.

Understanding overlap in overprescribing can help identify unique prescriber populations in which antibiotic and opioid stewardship can be focused and support aligned efforts to improve the use of these agents and reduce harm. Our objective was to evaluate the association between being a high prescriber of antibiotics and a high prescriber of opioids in the primary care setting.

Competing interests: None declared.

This article has been peer reviewed.

Correspondence to: Bradley Langford, bradley.langford @ oahpp.ca

CMAJ Open 2021. DOI:10.9778/cmajo.20200122 


\section{Methods}

\section{Study design and setting}

We performed a cross-sectional study evaluating primary care physician prescribers of opioids and antibiotics between Mar. 1, 2017, and Feb. 28, 2018. We conducted the study using databases held at ICES in Ontario, Canada's most populous province. ICES is an independent, nonprofit research institute whose legal status under Ontario's health information privacy law allows it to collect and analyze health care and demographic data, without consent, for health system evaluation and improvement. ICES is considered a prescribed entity under Ontario's Personal Health Information Protection Act. The institute securely collects, stores and analyzes personal health information for the 14 million residents of the province.

\section{Population}

Eligible physicians included all family medicine, general practice and community medicine physicians in Ontario. We excluded physicians with fewer than 500 patient visits per year ( $<2$ visits per business day, representative of less active clinicians), physicians prescribing the lowest $5 \%$ of antibiotic prescriptions and those prescribing the lowest $5 \%$ of opioid prescriptions to ensure that only active prescribers were included in the cohort. We also excluded physicians with a focus or specialty of pain ( $\geq 20$ billings for pain management) or palliative care ( $\geq 25 \%$ of all billings for a palliative care indication) to ensure that high opioid prescribers were not categorized as such because of their unique practice setting and patient populations.

\section{Data sources}

Antibiotic prescribing was identified with the use of Xponent, an outpatient medication database owned by IQVIA, a thirdparty company and a common source of global outpatient prescribing data. This database includes dispensed outpatient medications aggregated at the physician level. We included orally administered antibiotics in the World Health Organization Anatomical Therapeutic Chemical Classification J01.9 A list of included antibiotic classes is shown in Appendix 1 (available at www.cmajopen.ca/content/9/1/E175/suppl/DC1). Only new (nonrepeat and nonrefill) antibiotic prescriptions were included. Xponent captures $61 \%$ of medications dispensed from 2187 of 4391 community pharmacies in the province, and a patented, internally validated geospatial projection algorithm extrapolates these data to estimate $100 \%$ of prescribing by physicians..$^{10}$ We previously conducted a validation analysis in patients aged 65 years or more, which showed that this database has reasonable performance (specificity of $92.4 \%$ and positive predictive value of $77.2 \%$ ) in identifying the top quartile of antibiotic-prescribing physicians when compared to the Ontario Drug Benefit database, the province's highly accurate outpatient drug-dispensing database. ${ }^{11}$

We identified opioid prescribing using the province's Narcotics Monitoring System, which collects patient-level dispensing data for all controlled drugs (including opioids) from all outpatient pharmacies in Ontario. ${ }^{12}$ All opioid-containing products within the Narcotics Monitoring System were captured regardless of indication with the exception of opioids used primarily for managing opioid use disorder (methadone and buprenorphine-naloxone). A list of included opioids is shown in Appendix 2 (available at www.cmajopen.ca/ content/9/1/E175/suppl/DC1).

Both Xponent and the Narcotics Monitoring System include unique identifiers for each physician, allowing data to be linked with ICES databases. Ontario has a universal health care system that provides publicly funded access to hospital and physician services to all citizens, permanent residents and certain refugees. Population-wide ICES databases included the Registered Persons Database (which contains demographic information such as date of birth, date of death and sex), the Ontario Health Insurance Plan database (which contains all physician billing information, including patient visits), the ICES Physician Database (which contains demographic information on all licensed Ontario physicians) and the Canadian Institute for Health Information Discharge Abstract Database and National Ambulatory Care Reporting System, which contain information related to hospital admissions and emergency department visits, respectively. At the physician level, we also captured long-term care visits, emergency department visits as a proportion of all visits and patient roster size.

\section{Outcomes}

We measured the main primary and secondary outcomes at the prescriber level as 6 separate metrics: initiation, selection and duration of antibiotics, and initiation, selection and duration of opioids. Based on our previous validation study ${ }^{11}$ and the proportion of estimated outlier prescribers in previous studies, ${ }^{13-15}$ we considered physicians ranked in the top quartile of any of these metrics to be high prescribers for that particular metric.

\section{Primary outcomes}

Primary outcomes included initiation of antibiotic therapy and initiation of opioid therapy. Antibiotic initiation was defined as a physician's antibiotic prescription volume (number of prescriptions) divided by his or her patient visits to determine the antibiotic initiation rate. This rate of prescribing is highly variable, even after patient characteristics are accounted for, ${ }^{16}$ and is strongly correlated with estimated unnecessary antibiotic prescribing (Pearson's $r=0.93$ ). ${ }^{17} \mathrm{Opi}$ oid initiation was estimated as the volume of opioid prescriptions initiated by a prescriber. We calculated it by dividing the number of patients with 1 or more opioid prescriptions written by a given physician by the number of patients seen by that physician during the study period.

\section{Secondary outcomes}

Secondary outcomes included antibiotic selection and duration of treatment, and opioid selection and duration of treatment. We defined antibiotic selection as the proportion of all antibiotics prescribed that were broad-spectrum; 
broad-spectrum antibiotic classes included penicillin with $\beta$-lactamase inhibitor, fluoroquinolones, macrolides, secondand third-generation cephalosporins, and clindamycin. This definition is based on previous studies of broad-spectrum prescribing and identified risk of community-acquired Clostridium difficile infection. ${ }^{18,19}$

We defined opioid selection as the proportion of a physician's patients prescribed an opioid who received a prescription for 1 or more high-dose opioids, defined as any prescription with a dispensed daily dosage greater than 90 morphine milligram equivalents. The 2017 Canadian Guideline for Opioids for Chronic Non-Cancer Pain ${ }^{20}$ and the CDC [Centers for Disease Control and Prevention] Guideline for Prescribing Opioids for Chronic Pain - United States, 2016 ${ }^{21}$ recommend avoiding increasing dosages beyond 90 morphine milligram equivalents or carefully justifying any decisions to titrate to this dose.

We defined duration of antibiotic treatment as the proportion of all antibiotic prescriptions that were prescribed for a duration of longer than 8 days. We selected this threshold because most uncomplicated infections managed in primary care settings require a duration of antibiotic therapy of 7 days or less. ${ }^{22}$ We defined duration of opioid treatment as the proportion of a physician's patients prescribed an opioid who had 1 or more opioid prescriptions with a dispensed duration longer than 28 days. The CDC guideline recommends limiting initial opioid treatment for acute pain to 7 days. ${ }^{21}$ The probability of continuing on a long-term $(\geq 1 \mathrm{yr})$ opioid increases substantially when the first prescription supply exceeds 10 or 30 days. ${ }^{23}$ We used a conservative estimate of 28 days to identify prescribers who select longer courses of opioid therapy.

\section{Statistical analysis}

We compared the individual and practice characteristics of physicians in the bottom $75 \%$ (nonhigh prescriber) and top $25 \%$ (high prescriber) of antibiotic and opioid initiation categories. We calculated a $\kappa$ statistic with $95 \%$ confidence intervals (CIs) to determine the agreement between high antibiotic and high opioid initiation and high prescribing in at least 2 metrics (e.g., initiation, selection and duration). We categorized $\kappa$ values according to the extent of agreement between metrics: 0 to 0.20 (none to slight), 0.21 to 0.40 (fair), 0.41 to 0.60 (moderate), 0.61 to 0.80 (substantial) or 0.81 to 1.00 (almost perfect). ${ }^{24}$ Analyses were carried out with SAS Enterprise Guide version 7.1 (SAS Institute).

\section{Ethics approval}

This project received approval from Public Health Ontario's Ethics Review Board.

\section{Results}

During the study period, there were 13102 potentially eligible physicians, of whom 3108 were excluded: 11 were not linkable to ICES databases, 976 had fewer than 500 patients visits over the study period, 161 were pain specialists, 221 were palliative care specialists, 587 were among the lowest $5 \%$ of opioid prescribers, and 1152 were among the lowest $5 \%$ of antibiotic prescribers. The median number of daily patient visits per physician for the 9994 physicians included was 18 (interquartile range [IQR] 14-25).

Antibiotic- and opioid-prescribing metrics varied across physicians. Antibiotics were initiated in a median of $8.9 \%$ (IQR $6.0 \%-13.1 \%$ ) of visits. Broad-spectrum antibiotics were selected in $40 \%$ (IQR $31 \%-51 \%$ ) of antibiotic prescriptions. The proportion of long-term antibiotic prescriptions was $32 \%$ (IQR 21\%-46\%).

The median proportion of patients with an opioid initiated during the study period was 5.7\% (IQR 3.4\%-9.1\%). Highdose opioids were selected in $6.1 \%$ (IQR $2.0 \%-10.9 \%$ ) of prescriptions, and the proportion of prolonged-duration opioid prescriptions was $27 \%$ (IQR $11 \%-41 \%$ ).

\section{High antibiotic prescribers}

Compared to nonhigh prescribers of antibiotics, high antibiotic initiators tended to work in busier practices with a greater proportion of patient visits per day, were more likely to be male and had a greater proportion of visits that were emergency department visits (Table 1).

There was a negligible to weak association between the different antibiotic-prescribing metrics: antibiotic initiationantibiotic selection, $\kappa=0.05,95 \%$ CI 0.03 to 0.07 ; antibiotic initiation-antibiotic duration, $\kappa=0.06,95 \%$ CI 0.04 to 0.08 ; and antibiotic selection-antibiotic duration, $\kappa=0.06,95 \%$ CI 0.04 to 0.08 .

\section{High opioid prescribers}

Compared to nonhigh prescribers of opioids, high opioid initiators were more likely to be male, be in late career, have more nursing home patient visits, have a greater proportion of patients aged 65 years or more, and have a greater number of patient visits when (Table 1).

There was slight agreement between the different opioidprescribing indices: opioid initiation-opioid selection, $\kappa=$ $0.16,95 \%$ CI 0.14 to 0.18 ; opioid initiation-opioid duration, $\kappa=0.15,95 \%$ CI 0.13 to 0.17 ; and opioid selection-opioid duration, $\kappa=0.18,95 \%$ CI 0.16 to 0.20 .

\section{High prescribers of both antibiotics and opioids}

The $\kappa$ coefficient between high antibiotic initiators and high opioid initiators was 0.00 (95\% CI -0.02 to 0.02$)$, indicating there was a lack of agreement between these 2 groups and the overlap between them was no greater than that due to chance alone (Table 2). A small subset of all physicians (618 [6.2\%]) were both high antibiotic initiators and high opioid initiators. There was low agreement between the other antibiotic- and opioid-prescribing metrics (Table 2).

\section{Interpretation}

Our study of more than 9000 primary care physicians indicated that there was a lack of correlation between being a high antibiotic prescriber and being a high opioid prescriber when various facets of prescribing (initiation, selection and 
Table 1: Characteristics of high and lower antibiotic and opioid initiators and their practices

\begin{tabular}{|c|c|c|c|c|}
\hline \multirow[b]{2}{*}{ Characteristic } & \multicolumn{2}{|c|}{ Antibiotic initiators; no. (\%) of physicians* } & \multicolumn{2}{|c|}{ Opioid initiators; no. (\%) of physicians* } \\
\hline & $\begin{array}{l}\text { Lowest } 75 \% \\
n=7496\end{array}$ & $\begin{array}{l}\text { Highest } 25 \% \\
n=2498\end{array}$ & $\begin{array}{l}\text { Lowest } 75 \% \\
n=7496\end{array}$ & $\begin{array}{l}\text { Highest } 25 \% \\
n=2498\end{array}$ \\
\hline \multicolumn{5}{|l|}{ Physicians } \\
\hline \multicolumn{5}{|l|}{ Median no. of daily patient visits } \\
\hline$<10$ & $528(7.0)$ & $194(7.8)$ & $494(6.6)$ & $228(9.1)$ \\
\hline $10-20$ & $3746(50.0)$ & $1068(42.8)$ & $3814(50.9)$ & $1000(40.0)$ \\
\hline$>20$ & $3222(43.0)$ & $1236(49.5)$ & $3188(42.5)$ & $1270(50.8)$ \\
\hline Age, median (IQR), yr & $53(44-61)$ & $53(44-61)$ & $51(42-59)$ & $58(49-66)$ \\
\hline \multicolumn{5}{|l|}{ Sex } \\
\hline Female & $3533(47.1)$ & $933(37.3)$ & $3784(50.5)$ & $682(27.3)$ \\
\hline Male & $3963(52.9)$ & $1565(62.7)$ & $3712(49.5)$ & $1816(72.7)$ \\
\hline \multicolumn{5}{|l|}{ Years since medical graduation } \\
\hline$<11$ & $1644(21.9)$ & $482(19.3)$ & $1830(24.4)$ & $296(11.8)$ \\
\hline $11-24$ & $2173(29.0)$ & $767(30.7)$ & $2410(32.2)$ & $530(21.2)$ \\
\hline$>24$ & $3679(49.1)$ & $1249(50.0)$ & $3256(43.4)$ & $1672(66.9)$ \\
\hline \multicolumn{5}{|l|}{ Country of medical school } \\
\hline Canada/United States & $4661(62.2)$ & $1527(61.1)$ & $4525(60.4)$ & $1663(66.6)$ \\
\hline Other & $1703(22.7)$ & $620(24.8)$ & $1657(22.1)$ & $666(26.7)$ \\
\hline Unknown & $1132(15.1)$ & $351(14.0)$ & $1314(17.5)$ & $169(6.8)$ \\
\hline \multicolumn{5}{|l|}{ Rurality } \\
\hline Urban & $6791(90.6)$ & $2296(91.9)$ & $6891(91.9)$ & $2196(87.9)$ \\
\hline Rural & $705(9.4)$ & $202(8.1)$ & $605(8.1)$ & $302(12.1)$ \\
\hline $\begin{array}{l}\text { Emergency department visits, mean } \\
\pm \mathrm{SD}, \%\end{array}$ & $8.35 \pm 22.6$ & $20.9 \pm 35.1$ & $14.03 \pm 29.3$ & $3.85 \pm 14.8$ \\
\hline Long-term care visits, mean $\pm \mathrm{SD}, \%$ & $2.44 \pm 9.9$ & $1.59 \pm 8.7$ & $1.03 \pm 4.8$ & $5.81 \pm 15.2$ \\
\hline Roster size, median (IQR) & $1101(752-1540)$ & $1080(616-1561)$ & $1026(683-1448)$ & $1303(893-1729)$ \\
\hline \multicolumn{5}{|l|}{ Practices } \\
\hline \multicolumn{5}{|l|}{ Age group, mean $\pm S D$, yr } \\
\hline$<18$ & $15.6 \pm 7.1$ & $17.7 \pm 7.0$ & $17.1 \pm 7.1$ & $131 \pm 6.4$ \\
\hline $18-64$ & $60.5 \pm 10.0$ & $61.3 \pm 9.6$ & $62.0 \pm 9.0$ & $56.7 \pm 11.3$ \\
\hline$\geq 65$ & $24.0 \pm 12.4$ & $21.0 \pm 11.4$ & $20.9 \pm 10.2$ & $30.2 \pm 14.7$ \\
\hline Female sex, mean $\pm \mathrm{SD}, \%$ & $57.1 \pm 9.1$ & $56.1 \pm 6.9$ & $57.7 \pm 8.5$ & $54.1 \pm 8.2$ \\
\hline \multicolumn{5}{|c|}{ Neighbourhood income quintile of patient population } \\
\hline Low (1-3) & $6141(81.9)$ & $2050(82.1)$ & $5942(79.3)$ & $2249(90.0)$ \\
\hline High (4-5) & $1355(18.1)$ & $448(17.9)$ & $1554(20.7)$ & $249(10.0)$ \\
\hline \multicolumn{5}{|c|}{ Comorbidities in previous $2 \mathrm{yr}$, mean $\pm \mathrm{SD}, \%$} \\
\hline Acute myocardial infarction & $0.5 \pm 0.5$ & $0.5 \pm 0.4$ & $0.5 \pm 0.4$ & $0.7 \pm 0.6$ \\
\hline Congestive heart failure & $0.9 \pm 0.9$ & $0.8 \pm 0.7$ & $0.8 \pm 0.8$ & $1.1 \pm 1.1$ \\
\hline $\begin{array}{l}\text { Chronic obstructive pulmonary } \\
\text { disease }\end{array}$ & $1.2 \pm 0.8$ & $1.1 \pm 0.7$ & $1.1 \pm 0.7$ & $1.4 \pm 0.9$ \\
\hline Diabetes & $1.6 \pm 0.8$ & $1.4 \pm 0.6$ & $1.4 \pm 0.7$ & $1.7 \pm 0.8$ \\
\hline Hypertension & $2.3 \pm 0.9$ & $2.1 \pm 0.8$ & $2.2 \pm 0.8$ & $2.4 \pm 1.0$ \\
\hline Asthma & $0.9 \pm 0.5$ & $1.1 \pm 0.5$ & $1.0 \pm 0.5$ & $0.8 \pm 0.5$ \\
\hline Mental health condition & $20.6 \pm 7.8$ & $20.8 \pm 6.2$ & $20.6 \pm 6.9$ & $20.8 \pm 8.9$ \\
\hline \multicolumn{5}{|c|}{ Health care use in previous $2 \mathrm{yr}$, mean $\pm \mathrm{SD}, \%$} \\
\hline Hospital admission & $14.6 \pm 6.3$ & $15.0 \pm 6.0$ & $14.4 \pm 5.8$ & $15.6 \pm 7.3$ \\
\hline Emergency department visit & $41.9 \pm 12.2$ & $46.1 \pm 13.7$ & $42.7 \pm 12.8$ & $43.8 \pm 12.5$ \\
\hline
\end{tabular}




\begin{tabular}{|c|c|c|c|}
\hline \multirow[b]{2}{*}{ Opioid } & \multicolumn{3}{|c|}{ Antibiotic; $\kappa(95 \% \mathrm{Cl})^{*}$} \\
\hline & Initiation & Selection & Duration \\
\hline Initiation & $0.00(-0.02$ to 0.02$)$ & $0.16(0.14$ to 0.19$)$ & 0.05 (0.03 to 0.07$)$ \\
\hline Selection & $-0.09(-0.11$ to -0.07$)$ & $-0.02(-0.04$ to 0.00$)$ & 0.03 (0.01 to 0.05$)$ \\
\hline Duration & $-0.09(-0.11$ to -0.07$)$ & 0.03 (0.01 to 0.05$)$ & 0.07 (0.05 to 0.09$)$ \\
\hline \multicolumn{4}{|c|}{$\begin{array}{l}\text { Note: } \mathrm{Cl}=\text { confidence interval. } \\
\text { "Values between }-0.01 \text { and }-0.20 \text { indicate slight disagreement, values including } 0 \text { indicate no agreement, and values between } 0.01 \\
\text { and } 0.20 \text { indicate slight agreement. }\end{array}$} \\
\hline
\end{tabular}

duration) were considered. This finding indicates that high antibiotic prescribers and high opioid prescribers tended to be different, with minimal overlap between the 2 groups. This suggests key differences in patient populations and drivers for antibiotic and opioid overuse.

Other studies have shown associations between prescribing of antibiotics and of other medications. Quinn and colleagues ${ }^{25}$ conducted a population-based cohort study of nursing home physicians, also in Ontario, and found that highintensity antibiotic prescribers were more likely than average-intensity antibiotic prescribers to also prescribe proton-pump inhibitors, benzodiazepines and opioids. They defined high prescribers as those who prescribed above the upper limit of 2 standard deviations based on the proportions of their patients who received a prescription for 1 of these medications. Seventeen percent of physicians in their study were classified as high antibiotic prescribers, whereas, in our study, we defined a high prescriber as being in the top $25 \%$. These differences in definitions and methodology, as well as the more narrow and defined population of long-term care prescribers, may account for the differences in findings between their study and ours.

Similarly, $\mathrm{Li}$ and colleagues ${ }^{26}$ evaluated primary care practices in the United Kingdom and found that antibiotic prescribing was associated with prescribing of other medications; the association persisted after adjustment for practice and patient characteristics. They defined prescribing by the number of prescriptions for a given medication divided by the practice size. However, they did not specifically explore the association between antibiotic prescribing and opioid prescribing. They concluded that the propensity to prescribe antibiotics is linked to the propensity to prescribe medications in general, which suggests that antimicrobial stewardship interventions should address broader prescribing behaviours beyond knowledge of appropriate antibiotic use.

Our study also differs from previous analyses in that we evaluated 3 facets of antibiotic and opioid prescribing: initiation, regimen selection and treatment duration. Daneman and colleagues $^{13}$ evaluated antibiotic prescribers in nursing homes and found that prescribing behaviour differed substantially across these 3 domains of prescribing. Although prescribing tendencies remained consistent over time for each prescriber, similar to our findings, there was weak to no correlation between the domains of initiation, selection and duration of antibiotics prescribed by primary care physicians. This suggests that strategies to optimize prescribing should be tailored to the specific prescribing behaviour of interest.

Our findings may be useful for public health professionals, family physicians, and opioid and antibiotic stewards seeking to develop strategies and identify efficiencies in addressing high-prescribing practices.

\section{Limitations}

Limitations to this work include the potential for unmeasured confounding characteristics in prescribers and their patient population. Since high opioid prescribers and high antibiotic prescribers tend to work in different practice settings, these differences may contribute to less overlap than would otherwise be expected. The Canadian Opioid Prescribing Guideline for Chronic Non-Cancer Pain ${ }^{20}$ was released in 2017, during our study period, and may have shifted practice patterns toward reduced opioid use, particularly for physicians who were previously high prescribers. If so, this may have masked any overlap that might have existed previously between high opioid prescribers and antibiotic prescribers. In fact, abrupt discontinuation of opioids represents inappropriate prescribing, as this can lead to withdrawal symptoms. ${ }^{27}$

It is also important to note that, although we selected 6 separate metrics of antibiotic and opioid prescribing as proxy measures of appropriateness, this does not equate to a definitive assessment of appropriateness. This is important since opioid and antibiotic stewardship efforts aim to optimize the appropriate use of these medications rather than indiscriminately reduce their use. An additional limitation is that there is no standard definition of "high prescriber." We chose to use the top quartile in our study, as this has been previously measured and validated against other standard metrics of antibiotic use. ${ }^{11}$

\section{Conclusion}

Among primary care physicians in Ontario, there was minimal overlap between those who were high antibiotic prescribers and those who were high opioid prescribers. Antibiotic and opioid stewardship strategies to address these public health crises require tailored approaches. 


\section{References}

1. Canadian antimicrobial resistance surveillance system report. Ottawa: Public Health Agency of Canada; 2020. Cat no HP37-21E-PDF. Available: https:// www.canada.ca/content/dam/hc-sc/documents/services/drugs-health-products/ canadian-antimicrobial-resistance-surveillance-system-2020-report/CARSS-2020 -report-2020-eng.pdf (accessed 2021 Feb. 17).

2. Antibiotic resistance: a global threat. Atlanta: Centers for Disease Control and Prevention; 2018. Available: https://www.cdc.gov/drugresistance/solutions -initiative/stories/ar-global-threat.html\#: :text=Antibiotic\%20resistance\%2C \%20when\%20germs\%20(i.e.,and\%2023\%2C000\%20deaths\%20per\%20year (accessed 2021 Jan. 13)

3. Scholl L, Seth P, Kariisa M, et al. Drug and opioid-involved overdose deaths - United States, 2013-2017. MMWR Morb Mortal Wkly Rep 2018;67:1419-27.

4. Ontario Agency for Health Protection and Promotion (Public Health Ontario); Office of the Chief Coroner; Ontario Forensic Pathology Service; Ontario Drug Policy Research Network. Opioid Mortality Surveillance Report: analysis of opioid-related deaths in Ontario Fuly 2017-fune 2018. Toronto: Queen's Printer for Ontario; 2019.

5. Gomes T, Khuu W, Martins D, et al. Contributions of prescribed and nonprescribed opioids to opioid related deaths: population based cohort study in Ontario, Canada. BMF 2018;362:k3207.

6. Dhalla IA, Mamdani MM, Gomes T, et al. Clustering of opioid prescribing and opioid-related mortality among family physicians in Ontario. Can Fam Physician 2011;57:e92-6.

7. Daneman N, Gruneir A, Newman A, et al. Antibiotic use in long-term care facilities. 7 Antimicrob Chemother 2011;66:2856-63.

8. Daneman N, Bronskill SE, Gruneir A, et al. Variability in antibiotic use across nursing homes and the risk of antibiotic-related adverse outcomes for individual residents. 7AMA Intern Med 2015;175:1331-9.

9. Guidelines for ATC classification and DDD assignment. Geneva: World Health Organization; 1996.

10. Boardman C. System and method for estimating product distribution using a product specific universe. US patent 7174304B1.2007 Feb. 6.

11. Schwartz KL, Chen C, Langford BJ, et al. Validating a popular outpatient antibiotic database to reliably identify high prescribing physicians for patients 65 years of age and older. PLoS One 2019;14:e0223097.

12. Narcotic monitoring system (NMS): pharmacy reference manual [version 1.2]. Toronto: Ontario Ministry of Health and Long-Term Care; 2012.

13. Daneman N, Campitelli MA, Giannakeas V, et al. Influences on the start, selection and duration of treatment with antibiotics in long-term care facilities. CMA7 2017;189:E851-60.

14. Daneman N, Gruneir A, Bronskill SE, et al. Prolonged antibiotic treatment in long-term care: role of the prescriber. FAMA Intern Med 2013;173:673-82.

15. Hallsworth M, Chadborn T, Sallis A, et al. Provision of social norm feedback to high prescribers of antibiotics in general practice: a pragmatic national randomised controlled trial. Lancet 2016;387:1743-52.

16. Schwartz KL, Brown KA, Etches J, et al. Predictors and variability of antibiotic prescribing amongst family physicians. I Antimicrob Chemother 2019;74: 2098-105.

17. Kitano T, Langford BJ, Brown KA, et al. The association between high and unnecessary antibiotic prescribing: a cohort study using family physician electronic medical records. Clin Infect Dis 2020 Aug. 12 [Epub ahead of print]. doi: 10.1093/cid/ciaa1139.

18. Steinman MA, Landefeld CS, Gonzales R. Predictors of broad-spectrum antibiotic prescribing for acute respiratory tract infections in adult primary care. 7AMA 2003;289:719-25.

19. Brown KA, Khanafer N, Daneman N, et al. Meta-analysis of antibiotics and the risk of community-associated Clostridium difficile infection. Antimicrob Agents Chemother 2013;57:2326-32.

20. Busse JW, Craigie S, Juurlink DN, et al. Guideline for opioid therapy and chronic noncancer pain. CMA7 2017;189:E659-66.

21. Dowell D, Haegerich TM, Chou R. CDC guideline for prescribing opioids for chronic pain - United States, 2016. 7AMA 2016;315:1624-45.

22. Spellberg B. The new antibiotic mantra - "shorter is better." JAMA Intern Med 2016;176:1254-5.

23. Shah A, Hayes CJ, Martin BC. Characteristics of initial prescription episodes and likelihood of long-term opioid use — United States, 2006-2015. MMWR Morb Mortal Wkly Rep 2017;66:265-9.
24. McHugh ML. Interrater reliability: the kappa statistic. Biochem Med (Zagreb) 2012;22:276-82.

25. Quinn KL, Campitelli MA, Diong C, et al. Association between physician intensity of antibiotic prescribing and the prescription of benzodiazepines, opioids and proton-pump inhibitors to nursing home residents: a populationbased observational study. 7 Gen Intern Med 2019;34:2763-71.

26. Li Y, Mölter A, White A, et al. Relationship between prescribing of antibiotics and other medicines in primary care: a cross-sectional study. Br 7 Gen Pract 2019;69:e42-51.

27. Pergolizzi JV Jr, Raffa RB, Rosenblatt MH. Opioid withdrawal symptoms, a consequence of chronic opioid use and opioid use disorder: current understanding and approaches to management. 7 Clin Pharm Ther 2020;45: 892-903.

Affiliations: Public Health Ontario (Langford, Chen, Daneman, Brown, Johnstone, Wu, Leung, Garber, Schwartz), Toronto, Ont.; Hotel Dieu Shaver Health and Rehabilitation Centre (Langford), St. Catharines, Ont.; ICES Central (Chen, Gomes, Schwartz); Sunnybrook Health Sciences Centre (Daneman); Dalla Lana School of Public Health (Brown, Schwartz), University of Toronto; Keenan Research Centre of the Li Ka Shing Knowledge Institute (Gomes), St. Michael's Hospital; Sinai Health System (Johnstone); Michael Garron Hospital (Leung), Toronto East Health Network, Toronto, Ont.; Ottawa Hospital Research Institute (Garber), Ottawa, Ont.

Contributors: Bradley Langford and Kevin Schwartz drafted the manuscript. Cynthia Chen and Kevin Brown analyzed the data. All of the authors contributed to the study conception and design, interpreted the data, revised the manuscript critically for important intellectual content, approved the final version to be published and agreed to be accountable for all aspects of the work.

Funding: This study was funded by the Physician Services Incorporated Foundation.

Data sharing: The data set from this study are held securely in coded form at ICES. Although data-sharing agreements prohibit ICES from making the data set publicly available, access may be granted to those who meet prespecified criteria for confidential access, available at https://www. ices.on.ca/DAS. The full data set creation plan and underlying analytic code are available from the authors on request, with the understanding that the programs may rely on coding templates or macros that are unique to ICES.

Disclaimer: This study was supported by ICES, which is funded by an annual grant from the Ontario Ministry of Health and Long-Term Care (MOHLTC). Parts of this material are based on data or information compiled and provided by the Canadian Institute for Health Information (CIHI). The opinions, results and conclusions reported in this paper are those of the authors and are independent from the funding sources. No endorsement by ICES, CIHI or the Ontario MOHLTC is intended or should be inferred.

Content licence: This is an Open Access article distributed in accordance with the terms of the Creative Commons Attribution (CC BYNCND 4.0) licence, which permits use, distribution and reproduction in any medium, provided that the original publication is properly cited, the use is noncommercial (i.e., research or educational use), and no modifications or adaptations are made. See: https://creativecommons.org/licenses/ by-nc-nd/4.0/.

Supplemental information: For reviewer comments and the original submission of this manuscript, please see www.cmajopen.ca/content/9/1/ E175/suppl/DC1. 